\title{
IMPORTANCIA DEL CONTROL Y LA EVALUACIÓN EN LA GESTIÓN PÚBLICA ECUATORIANA
}

\author{
IMPORTANCE OF CONTROL AND EVALUATION \\ IN DE ECUADORIAN PUBLIC MANAGEMENT
}

\author{
Liliana del Carmen Morillo Acosta', Nancy del Rocio Flores Hinojosa²
}

\begin{abstract}
Resumen
Este estudio se realiza con enfoque interpretativo en el marco de una metodología cualitativa; tiene como propósito fundamentar conceptualmente la importancia del control y la evaluación de la gestión pública como herramientas que coadyuvan a mejorar la calidad del desempeño de las instituciones públicas en su constante reto por erradicar el mal manejo de los recursos públicos e impulsar el logro de las metas fijadas en las agendas de gobierno. La exploración bibliográfica, complementada con el análisis de la experiencia ecuatoriana, en torno a la corrupción enfatiza que la eficiencia de los procesos se sustenta en los sistemas de control establecidos sobre la base de los valores organizacionales evidenciados en la transparencia de la rendición de cuentas; y, que como resultado de la evaluación se despliegan propuestas de cambio y mejoramiento de las políticas, programas y proyectos públicos.

Las enmiendas constitucionales a los artículos 211 y 212 aprobadas en diciembre de 2015 que eliminaron las competencias de la Contraloría General del Estado ecuatoriano para ejercer la evaluación del desempeño de la burocracia estatal y el logro de los objetivos institucionales, fueron derogadas en mayo de 2018; de cuyo análisis se concluye que, el control y la evaluación constituyen factores determinantes en el fortalecimiento y consolidación de los procesos de desarrollo institucional; y, que las recomendaciones vertidas como producto de su implementación, aportan en la formulación de políticas públicas y en la democratización de la gestión.
\end{abstract}

\section{Palabras clave}

Control, evaluación, gestión pública, rendición de cuentas, capacidades estatales.

\begin{abstract}
This study was performed with an interpretive approach within the framework of a qualitative methodology. The purpose of this study is to establish the importance of the control and the evaluation of public management as tools that help improve the performance of public institutions in their constant challenge of eradicating the mismanagement of public resources and promoting the accomplishment of the goals set in the government agendas. The literature review along with the analysis of the Ecuadorian experience around corruption emphasizes that the efficiency of the processes is sustained on the control systems established on the basis of the organizational values and evidenced in the transparency of the accountability. As a result of this evaluation, proposals for change and improvement of public policies, programs, and projects are presented.

The constitutional amendments to articles 211 and 212 approved in December 2015, eliminating the Ecuadorian State Comptroller General competencies to perform assessments on the state bureaucracy and the achievement of institutional objectives, were abolished in May 2018. As a result of this analysis, it is concluded that, the control and the evaluation are determining factors in the strengthening and consolidation of institutional development processes. The recommendations made as a result of the implementation in the control and evaluation, contribute to the formulation of public policies and the democratization of management.
\end{abstract}

\section{Keywords}

Control, evaluation, public management, accountability, state capacity.

JEL Classification: H11, H83 


\section{Introducción}

La lucha constante de las organizaciones estatales por combatir la corrupción, el mal uso de los recursos y el abuso del poder; además de la imperante preocupación por alcanzar los objetivos institucionales que se orientan a satisfacer las demandas sociales para cumplir con los intereses colectivos y comunes, son factores que conllevan a la implementación de sistemas de control y evaluación como herramientas efectivas que permitan en un tiempo deseado la identificación de desviaciones que limiten e impidan el logro de la visión y misión de las instituciones públicas. El empleo de métricas para evaluar la eficacia de una institución pública resulta más que la búsqueda de posicionamiento e institucionalidad, una necesidad por cumplir con su objetivo de servicio integral a la sociedad (Valencia et al., 2019, p. 12).

El escenario espacial de la presente investigación es de alcance nacional, siendo el Ecuador el país que constituye la base del análisis para determinar la imperante necesidad de que en la gestión pública se implementen adecuadamente los controles vigentes y se instituyan nuevos mecanismos que garanticen la utilización adecuada de los recursos.

Datos históricos demuestran que en Ecuador la corrupción y el despilfarro se han acentuado en los últimos 13 años. Pese a la bonanza económica más grande de la historia republicana alcanzada durante los dos períodos presidenciales del economista Rafael Correa, como resultado de los ingresos provenientes de la venta del petróleo, en el año 2020 se registran niveles exorbitantes de corrupción y fraudes llevados a cabo en medio de la crisis mundial resultado de la pandemia de coronavirus que provoca la enfermedad de COVID-19.

Según las autoras del presente documento, los controles y la administración pública están fuertemente articulados, los factores que originaron el surgimiento de la una es la consecuencia de la otra, por lo cual, profesionales especializados concibieron como sinónimos al control y a la administración. Así también, la dicotomía controlevaluación toma fuerza al constituirse como acciones secuenciales. La aplicabilidad de los controles garantiza eficiencia en la utilización de los recursos públicos, el estricto cumplimiento de los actos administrativos y la transparencia en la información promulgada a través de la rendición de cuentas ( $a c^{-}$ countability). En tanto que, la evaluación es el control posterior de las operaciones, creados con la finalidad de determinar el nivel de desempeño de los colaborares públicos y el cumplimiento de los procesos administrativos, operativos y financieros de las instituciones, los resultados obtenidos de la revisión constituyen importantes insumos para proceder, por un lado, a la rectificación de errores, identificación de responsables y determinación de sanciones; y, por otro, aportan a la formulación de propuestas de mejoramiento de la intervención realizada por la administración pública de manera directa, o bien a través de la ejecución de programas y proyectos para satisfacer las demandas sociales y la formulación de políticas públicas.

La aplicación del control y de la evaluación puede realizarse por varios caminos, en esta investigación se identificaron dos instrumentos de probada efectividad: el presupuesto y la auditoría de gestión. De acuerdo al enunciado de Asensio (2015) el presupuesto es el producto de la planeación de actividades y la valoración de las mismas, basándose en datos históricos y en la proyección de acciones que son parte de una agenda institucional que requieren del monitoreo y control de su ejecución. Por su parte, la auditoría de gestión constituye el examen profesional ejecutado por los organismos de control que son formal y legalmente cons- 
tituidos para este fin, que a través de su aplicación metodológica promueve la consolidación democrática y fomenta la transparencia en el desempeño individual de los colaboradores del Estado (Bolívar, 2012).

El objetivo de este documento es fundamentar conceptualmente con base en la exploración bibliográfica y el análisis de la realidad ecuatoriana, la importancia del control y la evaluación de la gestión pública como herramientas que promueven el mejoramiento de la calidad de los procesos institucionales, a partir de la detección, investigación y sanción por el mal uso de los recursos del Estado.

Las conclusiones vertidas como producto de su aplicación generan pautas para la rectificación de errores y aportan con información importante en la formulación de políticas públicas y procedimientos administrativos efectivos que garanticen el logro de los objetivos.

\section{Metodología}

De acuerdo a la conceptualización descrita en Hernández et al. (2010, p. 7), por la naturaleza y objetivo de la presente investigación, se considera apropiada la aplicación de una metodología cualitativa para lograr identificar las variables determinantes que marcan la necesidad de disponer de mecanismos de control de la gestión pública y la evaluación correspondiente. Para lo cual, se procedió a la revisión de documentación científica fundamentada en fuentes primarias que incluyen libros, artículos y documentos, que permitieron identificar la fundamentación teórica de la administración pública, los orígenes del control y de la evaluación de la gestión en el marco de los paradigmas burocrático y posburocrático, cuyo entendimiento aporta, tanto a la ubicación espacial y temporal de los conceptos como al reconocimiento de su conveniente implementación para fortalecer las estructuras administrativas del Estado, y así alcanzar eficiencia en la ejecución de programas y proyectos en el marco de las agendas institucionales, locales y nacionales.

Se participó en mesas de diálogo y conversatorios con grupos especializados de la academia para analizar la importancia de la implementación de controles y procesos de evaluación en la gestión pública en el marco de la experiencia vivida en Ecuador a efecto de las enmiendas constitucionales a los artículos 211 y 212, numeral 2 aprobadas en diciembre de 2015, con las cuales se retiró la competencia de la Contraloría General del Estado para la realización de las Auditorías de Gestión en las entidades gubernamentales; $y_{\text {, }}$ la incidencia en la derogación de las mentadas enmiendas a partir de mayo de 2018.

A partir de lo mencionado, y con el objetivo de fortalecer la evaluación a través de la Auditoría de Gestión, se analiza la metodología utilizada por la Contraloría General del Estado ecuatoriano vigente, y se proponen cambios basados en la experiencia docente y profesional de las autoras que combinan los criterios adoptados en el sector público (Guía metodológica para auditoría de gestión, 2011, pp. 12-25) y en el sector privado (Franklin, 2013, pp. 75-122).

Una de las razones que inspiraron la elaboración de este documento, es demostrar la importancia de la aplicabilidad de la evaluación como herramienta de modernización del Estado y no como una simple técnica administrativa, para el efecto, es importante reflexionar sobre el alcance de la evaluación, la identificación de los involucrados en el proceso (quiénes deben evaluar) y la utilización de los resultados obtenidos.

\section{Marco teórico}

\section{El control y la administración pública}

El contexto histórico del control es tan amplio como la administración pública misma, las iniciativas y tendencias que originaron el 
surgimiento de la una es la consecuencia de la existencia de la otra, revelándose una dependencia irrefutable y casi obsesiva entre ambas. Así, los primeros especialistas de la administración concibieron al control y a la organización como sinónimos, el control debía estar presente para garantizar responsabilidad y eficiencia en la estructura y diseño organizacionales.

Reconociendo que el paraguas de la gestión pública aborda los eslabones de las operaciones gubernamentales visibles en el planeamiento, organización, dirección, coordinación y control en la formulación e implementación de políticas públicas, surge el requerimiento de evaluar no solo las actividades realizadas, sino también el desempeño y el logro del irrefutable objetivo institucional de solucionar los problemas públicos, el mismo que se encuentra íntimamente relacionado con la agenda de gobierno.

En la antigüedad, los primeros indicios del surgimiento del control administrativo son atribuidos a la civilización sumeria en el año 5000 a. C., posteriormente, en los años 4000 y 2000 a. C., los egipcios, reconocen la necesidad de planear, organizar y regular; $y$, la coordinación de las acciones de sus gobernantes, respectivamente. Más adelante, en el año 1800 a. C., en Babilonia, con el Código de Hammurabi, aparece el control del comercio; y, en el 1600 a. C., nuevamente los egipcios revelan la necesidad de replantear la organización de la administración y el control del territorio que ejercía el Gobierno central.

Años después, alrededor del 1800 a. C., en Babilonia emerge el Código de Hammurabi, el que considera aspectos relevantes acerca del control del comercio. Con el paso del tiempo, en el año 1600 a. C., nuevamente los egipcios presentan la iniciativa de reorganizar la administración y el control de los territorios ejercido por el gobierno central.

Después, en China, durante el dominio de las dinastías Hsia o Hia, Shang, Zhou,
Mencius entre los años 1994 a. C. y 500 a. C. surgen las primeras prácticas que utilizan técnicas administrativas y de control en los gobiernos territoriales y queda manifiesta la conveniencia de disponer de sistemas y estándares que permitan medir el grado de cumplimiento de las acciones administrativas. Posteriormente, entre los años 618-917, cambia la estructura del gobierno imperial, para lo cual se emitieron leyes administrativas y penales. Luego, en Venecia, durante la época medieval, en el año 1436, los directores del Arsenal utilizaron controles contables, administraron los inventarios, incluyeron controles de costos y de personal para el manejo de su flota naval. Finalmente, en 1554, durante el marco administrativo imperial de la nueva España, se conformó la Junta Superior de Hacienda, encargándosele la función de atender las finanzas de la Colonia, instancia que con el tiempo colaboró con el Consejo de Finanzas y la Superintendencia de la Real Hacienda (Franklin, 2007, pp. 4, 5).

En 1900, con el emblemático aporte de Frederick Windslow Taylor en su obra The Principles of Scientific Management emerge el método científico para analizar el trabajo y propiciar la eficiencia en las actividades productivas, los fundamentos de esta tendencia son fortalecidos con los aportes de Herny L. Gantt en 1901 y de Harrington Emerson en 1910. En este escenario, la administración pública asigna especial interés en la comprensión de sus estructuras burocráticas y reconoce la importancia de implementar controles efectivos que conlleven a la eficiencia organizativa que posteriormente pueda ser evaluada con base en la determinación de estándares que permitan la aplicación de indicadores y por ende la medición del desempeño y cumplimiento de objetivos (Shafritz y Hyde, 1999, p. 60).

Resulta imperativo mencionar a Max Weber, comúnmente llamado el padre de la sociología moderna, quien al señalar como 
factores determinantes del paradigma burocrático: la división del trabajo, los niveles de mando, la jerarquía organizativa y la cadena de mando, los sistemas de información, la departamentalización y el desarrollo de reglas, medidas políticas y procedimientos en operación; y, el alcance del control, reconoce que ante el crecimiento de las estructuras organizacionales aumentaron las presiones internas que demandaron de estrategias y la implementación de un rigor metodológico organizacional en aras de mantener la disciplina y el comportamiento ético en el desempeño de las funciones encomendadas a los servidores públicos, para alcanzar como objetivo final la transparencia en la rendición pública de cuentas y de gestión (Shafritz y Hyde, 1999, pp. 60-61).

Desde el punto de vista del Estado, resulta ineludible que los fines propuestos se puedan alcanzar si las acciones que ejecuta no se desenvolvieran en un escenario regido por un sistema de control jurídico disciplinario, que incluya deberes, obligaciones con normas claras y un régimen disciplinario; así, "el principio de legalidad como elemento imprescindible de un estado de derecho, impone a la administración pública la exigencia del cumplimiento de la ley y le otorga facultades de actuación estableciendo límites..." (García de Enterría, 2001) citado en (Zambrano y Yokir, 2017, p. 156).

Miradas recientes, afirman que el control es transversal en las organizaciones, que si bien, la existencia de los sistemas de control es responsabilidad de la máxima autoridad, la implementación depende de todos los niveles de la organización. Se crean con la finalidad de viabilizar los procesos, normar la conducta de las personas y para lograr identificar en un tiempo deseado, posibles desviaciones, despilfarro y mal uso de los recursos económicos, materiales y humanos. Los controles facilitan la rendición de cuentas de los funcionarios públicos y evitan que la información financiera, operativa y administrativa que se difunda sea falsa y fraudulenta (Morillo y Viteri, 2017, pp. 3537). En la misma línea, y complementando esta percepción, se afirma que la eficiencia y efectividad que se alcanza cuando existe un sistema de control sólido en las instituciones, es factible alcanzar los propósitos de la administración y promueve la institucionalidad de los entes fomentando la legalidad, integridad y transparencia (Mendoza-Zamora et al., 2018, pp. 217, 235).

En la misma línea, se observa que el enfoque acerca de la acción de controlar es similar en los países de la Región Andina, definiéndola como "la herramienta esencial del sistema, por la cual el personal técnico de sus órganos conformantes, mediante la aplicación de las normas, procedimientos y principios que regulan el control gubernamental, efectúa la verificación y evaluación, objetiva y sistemática, de los actos y resultados producidos por la entidad en la gestión y ejecución de los recursos, bienes y operaciones institucionales" (Ley Orgánica del Sistema Nacional de Control y de la Contraloría General de la República, 2002, p. 9).

\section{La evaluación de la gestión pública}

Como es conocido por los especialistas e historiadores de la Administración Pública, recién desde mediados del siglo XX con la propagación de la teoría de la burocracia del sociólogo Alemán Max Weber, se hace notoria la teoría de la administración pública, cuyo principio relevante fue la economía y eficiencia, es decir satisfacer las necesidades de la comunidad al menor costo posible, siendo este el objetivo concebido desde la óptica de la reforma administrativa. No obstante, e independientemente de los notables valores que ostenta la administración pública como son: democracia, justicia, igual trato ciudadano, bienestar, salud, la eficiencia 
continúa siendo la mayor finalidad; $y$, de este precedente surge el requerimiento de la evaluar la gestión.

Desde la mirada de Crozier (1997, p. 3) a pesar de que el paradigma burocrático se construyó sobre el eje de la especialización, las relaciones entre los programas y los resultados fueron vagos. Las metas con frecuencia no fueron claras debido a la fragmentación de las enormes estructuras administrativas características del sistema, lo cual llevó a la práctica de envolventes procedimientos administrativos y al formalismo, perdiendo la eficiencia añorada.

A la luz de la postura de reconocidos especialistas (Rhodes, 1991; Kettle, 1997; Bresser Pereira, 1997) citados en Ospina (2001, p. 1) este patrón burocrático, fue una de las causales que dieron paso al paradigma de la Nueva Gestión Pública o Neo-Gerencialismo en Nueva Zelanda, sobre el cual fluye la evaluación de las actividades gubernamentales como un componente crítico de la transición a la reforma del sector público, cuyo fin primordial fue lograr la eficiencia en el logro de desafíos, retos y responsabilidad por lograr el bienestar social.

Es menester citar que el paradigma posburocrático determina directrices que coadyuvan en necesidades orientadas a una gestión por resultados, como las siguientes: el mejoramiento del desempeño de las organizaciones en la formulación e implementación de políticas y programas y proyectos; la aplicación imperativa de un sistema claro de rendición de cuentas, la definición de estrategias para reducir el tamaño del Estado; $y$, el establecimiento de coyunturas fuertes entre las diferentes instancias del Estado. Estas directrices marcan la imperante necesidad de evaluar la gestión pública para evitar que "la existencia de 'burocracias con intereses propios' puede llevar a políticas fiscales inadecuadas, a la captura regulatoria o al mal funcionamiento del mercado laboral, dañando los incentivos y generando importantes costes de eficiencia" (Onrubia et al., 2019, p. 121).

Al vincular la evaluación al quehacer público, desde el criterio especializado de Ricardo Uvalle Berrones, en su artículo El Control y la Evaluación en la Administración Pública, afirma que "la evaluación es un ejercicio técnico, institucional, público y político que reclama el trabajo de deliberación y argumentación para construir los acuerdos que definen tanto la agenda de criterios como los objetivos, procedimientos, tiempos y productos esperados" (Uvalle, 2004, p. 61); más adelante, especifica que "la evaluación pública debe concebirse como un conjunto articulado de políticas, decisiones y acciones que tienen como objetivo descubrir, categorizar y argumentar los aciertos o los errores cometidos por la administración pública" (p. 63) en el logro de los objetivos de los programas, valorando el impacto expresado en costos, beneficios y omisiones que determinen la eficiencia de la gestión; y, por ende, el grado de satisfacción de los ciudadanos respecto a la administración pública evaluada.

Desde otras perspectivas, la evaluación como un proceso objetivo y congruente, cuando, en realidad, la objetividad misma y la supuesta imparcialidad de los evaluadores pueden ser la causa fundamental de los problemas para obtener los resultados, luego, el mismo autor, inserta profundidad al tema y afirma que la eficiencia y la efectividad en la gestión pública representan "valores típicos de los estudios de evaluación, chocan a menudo con la actitud de quienes aplican las políticas, más preocupados por la capacidad profesional y el poder de decisión de los especialistas que diseñaron las políticas" (Subirats, 1995, pp. 14-15). En la misma línea, (Kornberger et al., 2017, p. 197), refiere que las afirmaciones de eficiencia, equidad, calidad, economía y mayor transparencia agrupadas solo serán posible evidenciarlas en la 
esfera pública si los actores las internalizan como principios cotidianos en los procesos que ejecuten.

El alcance de la evaluación de la gestión abarca a toda la organización o una parte de ella en la que se valora la eficacia de las políticas públicas y sus reformulaciones, la consistencia de las estructuras administrativas, la calidad de la toma de decisiones con mayor énfasis en los resultados alcanzados en la ejecución de programas y proyectos, que, en los insumos y medios utilizados durante los procesos realizados, permitiendo la valoración del impacto logrado en el grupo beneficiario. Desde otra mirada, la mentada evaluación es "un ejercicio técnico a ser una herramienta política en la medida que abarque los tres niveles de la acción social, el macro, meso y micro, y en la medida que genere capacidad gerencial para establecer conexiones entre estos niveles" (Ospina, 2001, p. 1).

Según la visión de las autoras de esta investigación, un aspecto importante en el estudio de la evaluación es identificar a los involucrados en el proceso de evaluación, es decir quiénes ejercen la acción de evaluar, para lo cual, se concibe a la evaluación como un proceso abierto, continuo y propositivo que es desarrollado por actores diversos con el interés de juzgar la gestión gubernamental y el buen uso de los recursos. Este rol fiscalizador, en general, se encuentra encabezado por la ciudadanía, los partidos políticos, organizaciones sociales y políticas, instituciones públicas y privadas; en particular, ejercen la evaluación pública formal, las instancias gubernamentales constituidas para el efecto, como las contralorías y otros entes fiscalizadores.
Finalmente, los resultados desplegados de la evaluación como herramienta de gestión, ayudan a los directivos a identificar y entender la calidad del desempeño individual y colectivo de los miembros de la organización, así como también a visualizar e interpretar los factores determinantes de las causas de los logros y los fracasos de la institución en el contexto del direccionamiento estratégico de la misma, con la finalidad de que el manejo acertado de la información obtenida les permita tomar decisiones acertadas y logren alcanzar la añorada excelencia del servicio público. Así, se aplica la evaluación con el objetivo de que la intervención de las estructuras administrativas con énfasis en el cumplimiento de las políticas públicas, sea cada vez más eficiente y se articule con los valores que buscan el mejoramiento de la calidad de vida de los ciudadanos.

Por la relevancia y pertinencia que presenta la perspectiva clara y definida de Stoffaës (1993) citada en Araya (2002, p. 19) "la evaluación no debe ser confundida con el control de gestión y debe apoyarse sobre una ética cuidadosamente definida: su frecuencia y su continuidad no son las mismas, los responsables de la evaluación no deben ser perpetuados: el recurso a los profesionales exteriores debe ser promovido".

Con base en los autores expuestos anteriormente, se puede sintetizar dos aportes del ejercicio de la evaluación a la eficiencia de la gestión pública:

a. Promueve la consolidación democrática, al lograr determinar los niveles de responsabilidad de los funcionarios públicos y su obligación de rendir cuentas $^{3}$ acerca de la utilización de

3 "La rendición de cuentas puede estar desprovista, sea de elementos informativos, argumentativos o punitivos, sin que necesariamente hablemos de expresiones deficientes de rendición de cuentas. Serán ejercicios estrechos, limitados, enfocados, pero por eso no necesariamente constituyen - como lo llamaría la jerga politológica - 'subtipos disminuidos' de rendición de cuentas" (Schedler, 2006, p. 165). En el desarrollo de las actividades de los funcionarios públicos y, fundamentalmente, de quienes representan a las instituciones estatales, es trascendental determinar las directrices que establecen los parámetros del reporte tanto de la gestión como de la administración de recursos que deben presentar a quienes son los verdaderos propietarios del poder y gestores de riqueza de las sociedades: los ciudadanos. 
los recursos económicos, estructurales y humanos, sin perjuicio de la evaluación conseguida a partir de la aplicación de indicadores enmarcados en el enfoque de la accountability"; 4 dado que dichos recursos son escasos y su administración inadecuada conllevaría al fracaso de la gestión pública (Quesada, 2017, p. 69).

b. Fomenta la transparencia en el desempeño de las actividades de colaboradores públicos, a través de la mejora en la calidad del servicio que brindan a la ciudadanía, lo que incide en el bienestar de la colectividad por medio de la satisfacción de sus necesidades. Complementando esta consideración Longo (2004) enfatiza que, la selección y contratación de servidores públicos, debe realizarse aplicando procedimientos claros, transparentes, en donde la meritocracia debe ser la base para la identificación del personal idóneo, de esta manera se puede alcanzar la tan anhelada eficiencia en la administración gubernamental y aporta a la lucha contra la corrupción.

"Dentro de la dinámica de la globalización económica, la evaluación puede servir para legitimar procesos que sirvan fundamentalmente a los intereses hegemónicos y por ello es necesario pensar alternativas para la evaluación desde enfoques más comprensivos y que busquen la posibilidad de la transformación" (Porras, 2001, p. 43). No menos cierto, es que el ejercicio que representa la evaluación de la gestión pública, contribuye con valiosas lecciones aprendidas a los ciudadanos, a las autoridades y a los equipos de expertos, quienes posteriormente contribuyen con conocimiento de causa para mejorar los procesos y se con- vierten en actores activos en el desarrollo e implementación de las políticas públicas.

\section{Instrumentos de evaluación de la gestión pública}

Para proceder a la formulación de instrumentos de evaluación, es preciso diferenciar entre la evaluación ext ant y la evaluación ex post, en donde, la primera hace referencia a las condiciones, los medios, identificación de responsables y tiempos disponibles para iniciar los actos que permiten la valoración de los hechos y actos futuros; y, la segunda hace referencia a la valoración de la calidad demostrada o no en el desempeño de las instituciones administrativas del gobierno; $y$, demuestran el nivel de capacidad que tienen para generar compromiso con el logro de los objetivos y metas (Uvalle, 2004).

Habiendo recorrido el camino del control y la evaluación, es preciso señalar instrumentos efectivos de evaluación que permitan visualizar de manera técnica y sustentada el nivel de cumplimiento de los procesos respecto al acercamiento a la misión y visión institucional, realizados por la administración pública, cuya información llega a la ciudadanía e instancias pertinentes a través de la práctica de la accountability. En el presente documento, debido a las características e incidencia que deben reunir las herramientas de evaluación, considera que son de probada valía, el presupuesto y la auditoría de gestión.

\section{El presupuesto}

El presupuesto "es un registro del pasado en cuanto a éxitos, fracasos, logros, negociaciones y compromisos sobre las asignaciones pasadas que se reflejan en los rubros incluidos en el mismo, el presupuesto es

$4 \quad$ "El término accountability refiere la necesidad de controlar el poder público por medio de mecanismos que obligan a los funcionarios a informar y justificar sus acciones, y que pueden ser objeto de sanción" (Ríos et al., 2014, p. 266). Además, para ampliar la perspectiva término, véase (Barreda, 2011, pp. 273-274). 
también una declaración acerca del futuro" (Asensio, 2015, p. 44).

En tal sentido, desde la mirada de destacados autores, el presupuesto es concebido como un instrumento para perseguir la eficiencia, basado en tres perspectivas principales: la primera, el enfoque de asignación eficiente de los recursos, la segunda, el desafío de mantener la dicotomía estabilidad-desarrollo; y la tercera, la posibilidad del surgimiento de posibles distorsiones al objetivo estabilizador del presupuesto (Asensio, 2015, p. 45).

En la misma línea de los autores mencionados y asignando un enfoque paralelo Bo- lívar (2012, p. 40), señala que la acción de programar implica entre otras acciones, asignar recursos para el logro de los objetivos; analizar alternativas de optimización de los mismos y vincular al presupuesto con el planeamiento organizacional, con la finalidad de no solo medir la eficiencia y eficacia ${ }^{5}$ a partir de la medición de los objetivos alcanzados en relación con los costos, sino también de llegar a la valoración de las consecuencias de su no realización. Pueden considerarse las funciones sistematizadas a continuación, de las cuales las cuatro primeras, pueden verse como tradicionales y las siguientes como modernas:

Figura 1. Funciones de los presupuestos

\begin{tabular}{|c|c|}
\hline Funciones & Caracterización \\
\hline Plan financiero & $\begin{array}{l}\text { Esquema de actividad financiera, presente en el proceso ingreso gasto del Es- } \\
\text { tado. Es la expresión financiera de la política. Se constituye en una herramienta } \\
\text { imperativa de utilización de los recursos. }\end{array}$ \\
\hline Plan administrativo & $\begin{array}{l}\text { Representa la determinación de qué actividades deben realizarse, cuándo y cómo } \\
\text { tienen que ejecutarse; y, a través de esto, se evaluarán los programas de acción. }\end{array}$ \\
\hline Instrumento legal & $\begin{array}{l}\text { Plan político cuantitativamente expresado y aprobado por el órgano representa- } \\
\text { tivo de interés popular. } \\
\text { Permite el ejercicio del control formal porque establece límites de ejecución del } \\
\text { gasto y demanda de la rendición de cuentas o accountability. }\end{array}$ \\
\hline $\begin{array}{l}\text { Balance preventivo o instru- } \\
\text { mento contable }\end{array}$ & $\begin{array}{l}\text { Refleja e informa con anticipación el resultado de la gestión pública, permitiendo } \\
\text { determinar si habrá superávit, déficit o equilibrio cuantitativo. Además, permite } \\
\text { establecer la relación entre el cumplimiento de los planes operativos y la ejecu- } \\
\text { ción económica. }\end{array}$ \\
\hline $\begin{array}{l}\text { Instrumento de política } \\
\text { económica }\end{array}$ & $\begin{array}{l}\text { El presupuesto es la herramienta más importante porque considera el empleo de } \\
\text { factores productivos, las fuentes y distribución de los ingresos y la incidencia de } \\
\text { los precios de acuerdo a factores externos, por lo cual, se convierte en el termó- } \\
\text { metro de la administración pública. }\end{array}$ \\
\hline Instrumento de planeamiento & $\begin{array}{l}\text { Permite precisar el plan de acción del Estado y los organismos que lo conforman, la } \\
\text { dirección en la que ejerce la incidencia económica y la rendición de los servicios. }\end{array}$ \\
\hline Otras funciones & $\begin{array}{l}\text { Desempeña varias funciones: se constituye en un instrumento de programación, } \\
\text { permite la medición del crecimiento o decrecimiento económico, es el medio por } \\
\text { el que se asignan los recursos, entre otras. }\end{array}$ \\
\hline
\end{tabular}

Elaboración propia, con base en Bolívar (2012, pp. 40-46).

5 La eficacia se define como "una concepción general, la eficacia o efectividad, consisten en lograr o alcanzar los objetivos propuestos, las metas programadas, o los resultados deseados"; y, la eficiencia, "refiere al óptimo aprovechamiento de los recursos para el logro de objetivos, considerando la calidad y el tiempo asignado; se obtiene comparando el monto de recursos consumidos frente a bienes o servicios producidos; es la medición del desempeño" (Guía metodológica para auditoría de gestión, 2011, pp. 6-7). No obstante, más allá de la eficiencia y eficacia de las operaciones, "el desempeño institucional es el arquetipo que denota cómo la administración pública es capaz de fortalecer la legitimidad de los resultados que es propia de los valores democráticos" (Uvalle, 2004, p. 46). 
Lo expuesto conlleva al reconocimiento de la importancia y valía del presupuesto como instrumento de rendición de cuentas y valoración del impacto de la gestión gubernamental manifiesta en la agenda del gobierno, desde el inicio de su formulación, durante la ejecución y finalmente, en la evaluación correspondiente. No obstante, desde la mirada de otros expertos (Gómez R., 2004 , p. 115) que refieren críticamente que "el presupuesto público no ha logrado ser la expresión cuantitativa del plan de desarrollo del gobierno local, y más bien se ha convertido en una camisa de fuerza cuyo único objetivo es el control del gasto".

\section{Discusión y resultados}

\section{El control de gestión en Ecuador}

El control de la gestión pública en Ecuador a sus inicios estuvo sustentado en la Ley Orgánica de Administración Financiera y Control (Loafyc) mediante el Decreto Supremo n. ${ }^{0}$ 1429 publicado en el Registro Oficial 337 de 16 de noviembre de 1977 y su Reglamento. Años después, el control gubernamental fue promulgado en la Ley 2002-13 de la Ley Orgánica de la Contraloría General del Estado, publicada en el Registro Oficial 0595 de 12 de junio de 2002.

La Constitución de la República, en el artículo 211, señala: "La Contraloría General del Estado es un organismo técnico encargado del control de la utilización de los recursos estatales, y la consecución de los objetivos de las instituciones del Estado y de las personas jurídicas de derecho privado que dispongan de recursos públicos". El alcance de las actividades a que hace referencia el mentado artículo, abarca a la auditoría financiera, auditoría administrativa y auditoría de gestión.

No obstante, mediante las enmiendas a los artículos 211 y 212, numeral 2 de la Constitución de la República aprobadas por la Asamblea Nacional el 3 de diciembre de
2015, se eliminó la competencia de la Contraloría General del Estado para ejecutar auditorías de gestión, afectando severamente el control gubernamental, limitando la verificación del cumplimiento de los objetivos de las instituciones estatales y la medición del desempeño de los funcionarios públicos.

Habiendo transcurrido casi tres años, con fecha 01 de agosto de 2018, mediante Sentencia n. ${ }^{\circ}$ 018-18-SIN-CC, la Corte Constitucional resolvió declarar la Inconstitucionalidad a 13 de las 15 enmiendas a la Constitución, aprobadas en diciembre de 2015, entre ellas, las correspondientes a los artículos que compete a este documento.

Referencias históricas evidencian que en Ecuador las acciones fraudulentas que involucran a altos funcionarios del gobierno han proliferado en los últimos trece años, que a juicio de las autoras, estas pudieron haberse minimizado con el ejercicio de las auditorías de gestión por parte del ente de control, cuya ejecución pudo haber validado oportunamente la efectividad de los controles e identificado los riesgos potenciales que enfrentan las instituciones del Estado ante la débil y escasa asertividad de las decisiones adoptadas por las autoridades de gobierno que llevaron al Ecuador al exorbitante endeudamiento público y el crecimiento del Estado a niveles inaceptables. Por lo expuesto, a continuación, se presenta el estudio de la auditoría de gestión como una herramienta efectiva de control.

\section{La auditoría de gestión}

En el rastreo bibliográfico realizado, se evidencian varias posturas, las cuales confluyen en el enfoque de que la Auditoría de Gestión resulta ser el "examen comprensivo y constructivo de la estructura organizativa de una Organización, pública o privada y de sus métodos de control, medios de operación y empleo que da a sus recursos humanos y materiales (Triana, 2010, p. 25). 
Como punto de referencia, a continuación, se presenta la definición textual de Auditoría de Gestión que se encuentra tipificada en el artículo 21 de la Ley de la Ley Orgánica de la Contraloría General del Estado ecuatoriano:

Art. 21.- La Auditoría de Gestión es la acción fiscalizadora dirigida a examinar y evaluar el control interno y la gestión, utilizando recursos humanos de carácter multidisciplinario, el desempeño de una institución, ente contable, o la ejecución de programas y proyectos, con el fin de determinar si dicho desempeño o ejecución, se está realizando, o se ha realizado, de acuerdo a principios y criterios de economía, efectividad y eficiencia. (Ley Orgánica de la Contraloría General del Estado, 2005, p. 7)

En el cuerpo legal citado, se determina con detalle. que la auditoría gubernamental abarca todos los procesos administrativos y examina el desempeño de los colaboradores públicos, textualmente señala que su objeto es "el proceso administrativo, las actividades de apoyo, financieras y operativas; la eficiencia, efectividad y economía en el empleo de los recursos humanos, materiales, financieros, ambientales, tecnológicos y de tiempo; $y$, el cumplimiento de las atribuciones, objetivos y metas institucionales" (Ley Orgánica de la Contraloría General del Estado, 2005, p. 7). En concordancia con este concepto, la guía metodológica para la auditoría de gestión emitida por la Contraloría General del Estado, señala:

Un examen sistemático y profesional, efectuado por un equipo multidisciplinario, con el propósito de evaluar la eficacia de la gestión de una entidad, programa, proyecto u operación, en relación con sus objetivos y metas; determinar el grado de economía y eficiencia en el uso de los recursos disponibles; medir la calidad de los servicios, obras o bienes ofrecidos; $y$, el impacto socio-económico derivado de sus actividades. (Guía metodológica para auditoría de gestión, 2011, p. 6)
Desde la mirada del sector privado, la Auditoría Administrativa (de Gestión) es "la revisión analítica total o parcial de una organización con el propósito de precisar su nivel de desempeño y perfilar oportunidades de mejora para innovar el valor y lograr una ventaja competitiva sustentable" (Franklin, 2013, p. 15). Al tenor de la postura señalada Amador (2008, pp. 33-34) plantea que en las actividades privadas, no existen diferencias entre la auditoría administrativa y la auditoría de gestión, puesto que ambas denotan el examen de la gestión administrativa de las empresas, develadas en la adopción de decisiones efectivas que orientan la consecución del direccionamiento estratégico de las mismas.

De lo expuesto, las autoras de esta investigación deducen que, la auditoría de gestión es el control posterior de los procesos públicos efectuado por un grupo multidisciplinario de profesionales, con la finalidad de determinar el porcentaje de cumplimiento de los procesos respecto a la visión, misión, objetivos, metas, políticas institucionales y determina la efectividad de las estrategias, a través de la aplicación de indicadores de gestión.

Para tener una visión panorámica del direccionamiento estratégico de la empresa o institución, se propone la siguiente matriz.

La auditoría de gestión es ejercida de manera formal por los organismos técnicos designados por el gobierno, a quienes se les encarga el control de la utilización de los fondos públicos y la verificación del logro de los objetivos de las instituciones del Estado y de las empresas privadas que ejecutan acciones con fondos públicos (Constitución de la República del Ecuador, 2018, art. 211).

De acuerdo a la Ley Orgánica de la Contraloría General del Estado, a las actividades de control, fiscalización y auditoría "se debe los principios constitucionales, y especialmente los de legalidad, responsa- 
bilidad financiera, transparencia, economía, eficiencia y eficacia, junto con los criterios de equidad, y, ética y las políticas de descen- tralización y desconcentración operativas" (Ley Orgánica de la Contraloría General del Estado, 2005, 3er. considerando).

Figura 2. Matriz de Direccionamiento Estratégico

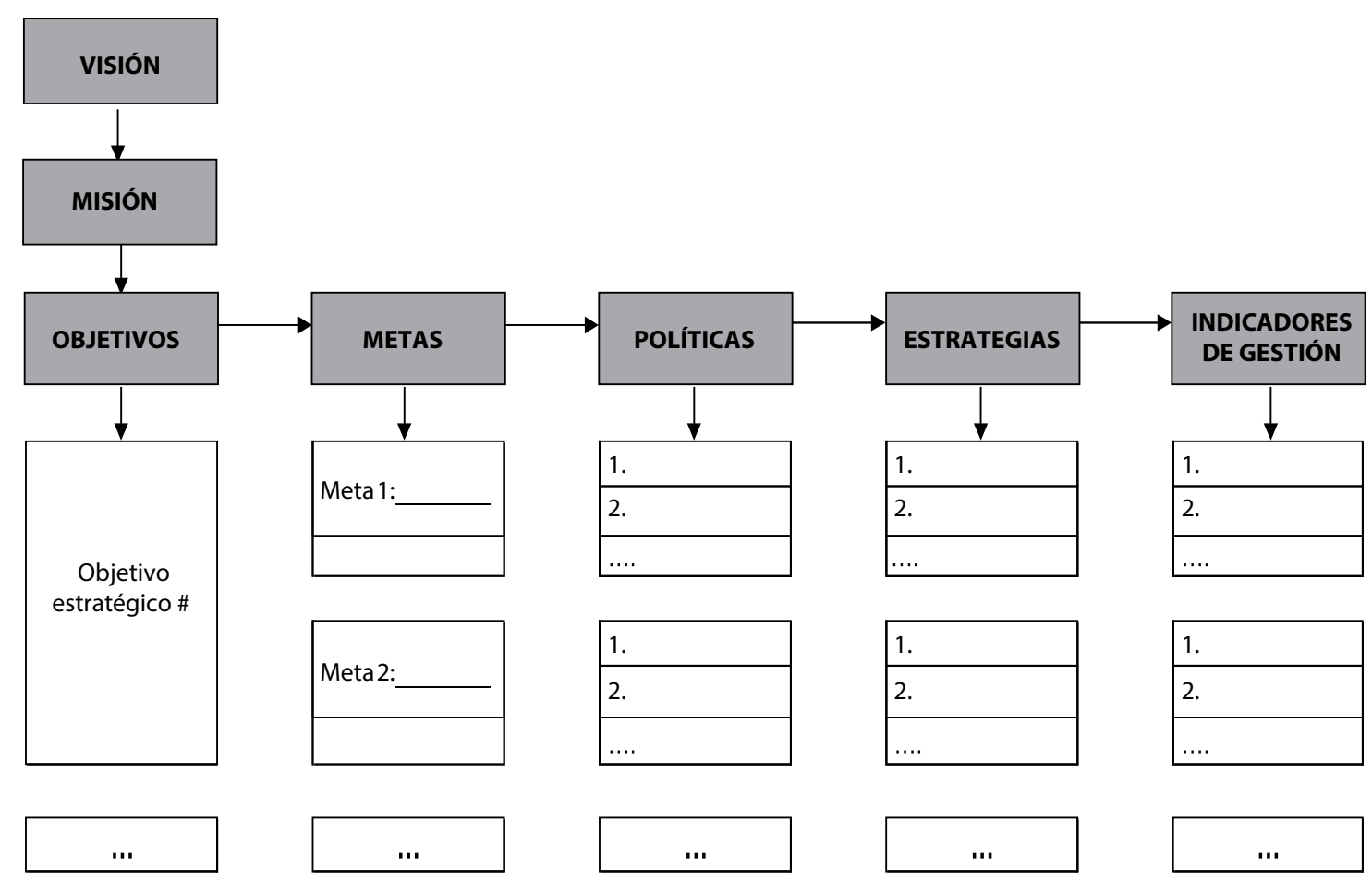

Elaboración propia.

La metodología sugerida a continuación para el ejercicio de la Auditoría de Gestión en el sector público, también puede ser adaptada al sector privado, se basa en el análisis de la normativa legal de creación de la entidad y que la faculta para su funcionamiento, seguida de cuatro fases diferenciadas, de cuya aplicación se obtendrá como producto final, un informe de gestión sustentado en evidencia suficiente, pertinente $y$ competente, como producto de la utilización de herramientas y técnicas profesionales.
La búsqueda de la eficiencia y eficacia en los procesos y acciones desarrolladas tanto en el sector público como en el sector privado, representan un desafío que se proponen alcanzarlo con base en la implementación de controles y posterior evaluación por medio de los indicadores de gestión, que además de lo señalado, permitirán valorar el impacto y la aceptación social que alcancen las acciones y servicios brindados a la comunidad. 
Figura 3. Metodología de la Auditoría de Gestión

\begin{tabular}{|c|c|c|}
\hline \multicolumn{3}{|c|}{ FORMALIZACIÓN DEL TRABAJO DE AUDITORÍA } \\
\hline INSTRUMENTOS LEGALES & $\begin{array}{l}\text { Definen los términos en que } \\
\text { se llevará a cabo la auditoría }\end{array}$ & $\begin{array}{l}\text { Orden de trabajo } \\
\text { Memorando de planificación }\end{array}$ \\
\hline \multicolumn{3}{|c|}{ APLICACIÓN DE LA METODOLOGÍA DE LA AUDITORÍA } \\
\hline FASE & PROCESOS & ACCIONES \\
\hline \multirow{2}{*}{ PLANIFICACIÓN } & Conocimiento institucional & $\begin{array}{l}\text { Recolección y análisis de información documental y } \\
\text { verbal proporcionada por la empresa }\end{array}$ \\
\hline & Programación & $\begin{array}{l}\text { Programa general } \\
\text { Programas especificos }\end{array}$ \\
\hline EJECUCIÓN & $\begin{array}{l}\text { Ejecución de los programas } \\
\text { específicos de auditoría }\end{array}$ & $\begin{array}{l}\text { Aplicación de técnicas y procedimientos de auditoría. } \\
\text { Recolección de evidencia suficiente y competente. } \\
\text { Elaboración de papeles de trabajo } \\
\text { Desarrollo de hallazgos }\end{array}$ \\
\hline \multirow[t]{2}{*}{ INFORME } & Informe preliminar & $\begin{array}{l}\text { Recepción y análisis de sustentos documentarios } \\
\text { para levantamiento o cambio del alcance de los ha- } \\
\text { llazgos encontrados }\end{array}$ \\
\hline & Informe final & Redacción del informe de resultados de la auditoría \\
\hline \multirow[t]{2}{*}{ SEGUIMIENTO } & $\begin{array}{l}\text { Cronograma de cumplimien- } \\
\text { to de recomendaciones }\end{array}$ & $\begin{array}{l}\text { Recepción y análisis de sustentos de cumplimiento de } \\
\text { recomendaciones }\end{array}$ \\
\hline & Informe de seguimiento & Redacción del informe de resultados de la auditoría \\
\hline
\end{tabular}

Elaboración propia, con base en Guía metodológica para auditoría de gestión (2011).

\section{Conclusiones}

- El control en las instituciones gubernamentales, constituye un medio y no un fin, es decir, acompaña técnicamente a la ejecución de los procesos, con el objetivo de que las acciones institucionales fluyan en coherencia con lo establecido en los planes y programas contenidos en las agendas de gobierno y en los lineamientos de las políticas públicas, en aras de alcanzar el objetivo final expresado en el bienestar de la colectividad.

- Resulta incuestionable la importancia de implementar sistemas de control sólidos en la gestión pública ecuatoriana, a partir de los hallazgos evidenciados en el crecimiento desmesurado de la corrupción y fraudes millonarios durante los últimos trece años, en que se han visto involucrados altos funcionarios del gobierno que han esquivando los controles que pese a ser abundantes, no ha demostrado ser efectivos.

- Del rastreo documental realizado en torno a la importancia de ejercer la evaluación de la gestión pública y del impacto vivido en Ecuador a efecto de las enmiendas constitucionales a los artículos 211 y 212 aprobadas en diciembre 03 de 2015 que derogaron la competencia de la Contraloría General del Estado para ejercer la supervisión del cumplimiento de los objetivos institucionales, ratifican que la auditoría de gestión es una herramienta que coadyuva al mejoramiento de la calidad de los procesos 
ejercidos por la administración pública, ofrece pautas importantes para la toma de decisiones y en caso de ser necesario, clarifica el escenario para la reorientación de las estrategias institucionales, toda vez que las conclusiones y recomendaciones derivadas del análisis, se levantan sobre evidencia suficiente, pertinente y competente.

- Del análisis de la metodología de la auditoría de gestión que se realiza en el sector público y de la auditoría administrativa en el sector privado, se llega a la determinación que, el control y la evaluación no son procesos excluyentes, son los medios que permiten alcanzar la efectividad de las acciones realizadas por las instituciones que conforman el aparato administrativo del gobierno. Por lo tanto, con la derogatoria de las enmiendas constitucionales realizada en mayo de 2018, mencionadas en el literal anterior, se ratifica que, el control y la evaluación deben ser reconocidos y revalorizados como instrumentos necesarios para que los sistemas de dirección, coordinación e implementación de las políticas públicas, programas y proyectos funcionen en favor de los beneficiarios de los mismos.

- Los sistemas de evaluación pública, contribuyen al fortalecimiento y consolidación de los procesos de desarrollo institucional, las conclusiones vertidas aportan para la formulación de políticas públicas y en la democratización de la gestión, puesto que los aprendizajes extraídos, revelan las fortalezas y debilidades identificadas en la administración del gasto público y de la administración pública.

- El presupuesto y la auditoría de gestión constituyen herramientas efectivas de evaluación de la gestión pú- blica, porque evidencian los aciertos, desaciertos y omisiones de la gestión pública; y, permiten la medición técnica de la gestión pública, identificando los cuellos de botella que retrasan el logro de los objetivos y promueven las rectificaciones necesarias para restablecer y/o construir las estructuras administrativas deseadas.

- El uso de indicadores de gestión, constituyen una herramienta importante, que, por un lado, permite monitorear el desempeño de los colaboradores de las instituciones; y por otro, visualiza el volumen de cumplimiento de los procesos organizacionales.

- Si queremos que las nuevas generaciones tengan un Ecuador viable, es indispensable redefinir el rol y tamaño del Estado.

\section{Referencias}

Amador, A. (2008). Auditoría administrativa. Proceso y aplicación. Mc. Graw Hill.

Araya, R. R. (2002). El contexto de la evaluación de proyectos sociales en el marco de la democracia deliberativa. Revista de Ciencias Sociales 17(30), 3-15.

Asensio, M. A. (2015). Economía fiscal. Presupuesto y finanzas estatales. Osmar D Buyatti-Librería Editorial. http://www.osmarbuyatti.com/Libros/ind_de_lib/2015/ Asensio_Economia_Fiscal.pdf

Barreda, M. (2011). La calidad de la democracia. Un análisis comparado de América Latina. Revista Política y Gobierno 18(2), 265-295.

Bolívar, M. A. (2012). El presupuesto público. Buyatti, Osmar D. Buyatti. http://www.osmarbuyatti.com/Libros/ind_de_lib/2012/ Bolivar\%20Finanzas\%20Publicas.pdf

Ley Orgánica del Sistema Nacional de Control y de la Contraloría General de la República, Pub. L. ñ. 27785, 44 (2002).

Ley Orgánica de la Contraloría General del Estado, Pub. L. n. ${ }^{0}$ 43, 35 (2005). https://www. 
oas.org/juridico/PDFs/mesicic5_ecu_ane_ cge_23_ley_org_cge.pdf

Constitución de la República del Ecuador. (2008). Decreto Legislativo 0. Registro Oficial 449, 20 de octubre. https://www.oas.org/juridico/pdfs/mesicic4_ecu_const.pdf

Guía metodológica para auditoría de gestión, Pub. L. No. Acuerdo n.․ 047-CG-2011, 43 (2011). https://es.scribd.com/document/ 394312923/Acuerdo047-Cg-2011guiametodoligicaparaauditoriadegestion

Franklin, E. B. (2007). Auditoría administrativa. Gestión estratégica del cambio (2. ${ }^{a}$ ed.). Pearson Educación. https://cucjonline. com/biblioteca/files/original/1a85f884ea 1f890592bdd5f941f5b715.pdf

Franklin, E. B. (2013). Auditoría administrativa. Evaluación y diagnóstico empresarial (3. ${ }^{a}$ ed.). Pearson Educación.

Gómez R., G. R. (2004). El presupuesto público en la gestión eficiente de los municipios. INNOVA, Revista de Ciencias Administrativas y Sociales, 24, 105-111. https://revistas. unal.edu.co/index.php/innovar/article/ view/25191

Hernández, R., Fernández, C., y Baptista, M. del P. (2010). Metodología de la investigación (5. ed.). Mc. Graw Hill. https:// www.esup.edu.pe/descargas/dep_investigacion/Metodologia $\% 20$ de $\% 20$ la\%20investigaci\%C3\%B3n\%205ta\%20 Edici\%C3\%B3n.pdf

Kornberger, M., Kornberger, R. E., Kornberger, C. y Höllerer, M. A. (2017). When Bureaucracy Meets the Crowd: Studying "Open Government" in the Vienna City Administration. Organization Studies, SAGE, 38(2), 179-200, 22. https://doi.org/DOI: 10.1177/0170840616655496

Mendoza-Zamora, W. M., García-Ponce, T. Y., Delgado-Chávez, M. I., y Barreiro-Cedeño, I. M. (2018). Internal control and its influence on the administrative management of the public sector. Revisa Cientifica Dominio de las Ciencias. 4(4), 206-240. http://dx.doi. org/10.23857/dom.cien.pocaip

Morillo, L., y Viteri, P. (2017). Auditoría Administrativa y de Gestión. Fundamentos metodológicos. Mengraf.
Onrubia, J., Pérez, J. J. y Sánchez-Fuentes, A. J. (2019). Public sector bureaucracies and economic growth. Revista de Economía Mundial, 51, 121-138.

Ospina, B. S. (2001). Evaluación de la gestión pública: Conceptos y aplicaciones en el caso latinoamericano. Revista del CLAD Reforma y Democracia, 19.

Vargas Porras, A. E. (2001). Enfoques evaluativos. Revista de Ciencias Sociales, 92-93, 35-45. https://www.revistacienciassociales.ucr. ac.cr/images/revistas/RCS92_93/03.pdf

Quesada, G. D. (2017). Importancia del enfoque transformador de la evaluación como ejercicio para orientar la construcción de políticas públicas. Revista Reflexiones, 96(1), 57-73. https://doi.org/10.15517/ rr.v96i1.30632

Ríos, R., Cortés Arbeláez, A., Suárez Valencia, M. C. y Fuentes Vélez, L. (2014). Accountability: Aproximación conceptual desde la filosofía política y la ciencia política. Colombia Internacional 82, 261-288. https://doi.org/dx.doi.org/10.7440/colombiaint82.2014,10

Schedler, A. (2006). ¿Qué es la rendición de cuentas? Foro Internacional XLVI-Reseñas, 2. https://www.redalyc.org/pdf/599/59918 310.pdf

Shafritz, J. M. y Hyde, A. C. (1999). Clásicos de la administración pública. Impresora y Encuadernadora Progreso.

Subirats, J. (1995). Los instrumentos de las políticas, el debate público y el proceso de evaluación. Revista de Gestión y Política Pública IV(1), 5-23.

Triana, R. L. (2010). La auditoría de gestión, una solución ante los retos provocados por los escándalos financieros. Escenarios 8(2), 21-30.

Uvalle, B. R. (2004). El control y la evaluación en la Administración Pública. Revista Mexicana de Ciencias Políticas y Sociales 46(192), 43-70. http://www.revistas.unam.mx/index.php/rmcpys/article/view/42463

Valencia, M. E., Pazmiño, G. E., Hurtado, K. del R., Ulloa, C. I., Scrich, R. J., Guerrero, N. F. y Scrich, A. J. (2019). Diseño de métricas para la evaluación de la eficacia mediante 
cuadros de mando en la dirección administrativa y financiera del sector público en Ecuador. Revista Espacios, 40(13. http:// www.revistaespacios.com/a19v40n13/ a19v40n13p30.pdf
Zambrano, R., y Yokir, M. (2017). El control a la gestión en la administración pública: Una mirada a las legislaciones de Ecuador y Perú. Revista San Gregorio 19, 154-167. 